\title{
Well-posedness for the generalized Benjamin-Ono equations with arbitrary large initial data in the critical space
}

\author{
Stéphane Vento, \\ Université Paris-Est, \\ Laboratoire d'Analyse et de Mathématiques Appliquées, \\ 5 bd. Descartes, Cité Descartes, Champs-Sur-Marne, \\ 77454 Marne-La-Vallée Cedex 2, France \\ E-mail: stephane.vento@univ-paris-est.fr
}

\begin{abstract}
We prove that the generalized Benjamin-Ono equations $\partial_{t} u+$ $\mathcal{H} \partial_{x}^{2} u \pm u^{k} \partial_{x} u=0, k \geq 4$ are locally well-posed in the scaling invariant spaces $\dot{H}^{s_{k}}(\mathbb{R})$ where $s_{k}=1 / 2-1 / k$. Our results also hold in the nonhomogeneous spaces $H^{s_{k}}(\mathbb{R})$. In the case $k=3$, local well-posedness is obtained in $H^{s}(\mathbb{R}), s>1 / 3$.
\end{abstract}

Keywords: NLS-like equations, Cauchy problem

AMS Classification: 35Q55, 35B30, 76B03, 76B55

\section{Introduction}

In this paper we pursue our study of the Cauchy problem for the generalized Benjamin-Ono equations

$$
\left\{\begin{array}{l}
\partial_{t} u+\mathcal{H} \partial_{x}^{2} u \pm u^{k} \partial_{x} u=0, \quad x, t \in \mathbb{R} \\
u(x, t=0)=u_{0}(x), \quad x \in \mathbb{R}
\end{array}\right.
$$

with $k$ an integer $\geq 3$ and with $\mathcal{H}$ the Hilbert transform defined via the Fourier transform by

$$
\mathcal{H} f=\mathcal{F}^{-1}(-i \operatorname{sgn}(\xi) \hat{f}(\xi)), \quad f \in \mathcal{S}^{\prime}(\mathbb{R}) .
$$

The Hilbert transform is a real operator, and consequently we look for real-valued solutions. In view of (1.1), we see that $\mathcal{H}$ is nothing but $-i$ 
on positive frequencies and $+i$ on negative ones. A very close equation to (gBO is then the derivative nonlinear Schrödinger equation

$$
\partial_{t} u-i \partial_{x}^{2} u \pm u^{k} \partial_{x} u=0 .
$$

for which all our results remain true. Furthermore, (gBO and (1.2) enjoy the same linear estimates, see Section 3 .

A remarkable feature of $(\mathrm{gBO})$ is the following scaling invariance: if $u(t, x)$ is a solution of the equation on $[-T,+T]$, then for any $\lambda>0$, $u_{\lambda}(t, x)=\lambda^{1 / k} u\left(\lambda^{2} t, \lambda x\right)$ also solves (gBO) on $\left[-\lambda^{-2} T,+\lambda^{-2} T\right]$ with initial data $u_{\lambda}(0, x)$ and moreover

$$
\left\|u_{\lambda}(\cdot, 0)\right\|_{\dot{H}^{s}}=\lambda^{s+\frac{1}{k}-\frac{1}{2}}\|u(\cdot, 0)\|_{\dot{H}^{s}} .
$$

Hence the $\dot{H}^{s}(\mathbb{R})$ norm is invariant if and only if $s=s_{k}=1 / 2-1 / k$ and we may expect well-posedness in $\dot{H}^{s_{k}}(\mathbb{R})$.

When $k=1$, $\mathrm{gBO}$ is the ordinary Benjamin-Ono equation derived by Benjamin [1] and later by Ono [15] as a model for one-dimensional waves in deep water. The Cauchy problem for the Benjamin-Ono equation has been extensively studied these last years, see [17, 16, 6]. In [18, Tao introduced a gauge transformation (a kind of Cole-Hopf transformation) which ameliorate the derivative nonlinearity, and get the well-posedness of this equation in $H^{s}(\mathbb{R}), s \geq 1$. Recently, combining a gauge transformation together with a Bourgain's method, Ionescu and Kenig [5] shown that one could go down to $L^{2}(\mathbb{R})$, which seems to be the critical space for the Benjamin-Ono equation. Note also that Burq and Planchon [4] have obtained well-posedness in $H^{s}(\mathbb{R}), s>1 / 4$ by similar methods. It is worth noticing that all these results have been obtained by compactness methods. On the other hand, Molinet, Saut and Tzvetkov [14] proved that, for all $s \in \mathbb{R}$, the flow map $u_{0} \mapsto u$ is not of class $\mathcal{C}^{2}$ from $H^{s}(\mathbb{R})$ to $H^{s}(\mathbb{R})$. Furthermore, building suitable families of approximate solutions, Koch and Tzvetkov proved in [11] that the flow map is actually not even uniformly continuous on bounded sets of $H^{s}(\mathbb{R}), s>0$. This explains why a Picard iteration scheme fails to solve the Benjamin-Ono equation in Sobolev spaces.

In the case of the modified Benjamin-Ono equation $(k=2)$, Kenig and Takaoka [10] have recently obtained the global well-posedness in the energy space $H^{1 / 2}(\mathbb{R})$. This have been proved thanks to a localized gauge transformation combined with a space-time $L^{2}$ estimate of the solution. It is 
important to note that this result is far from that given by the scaling index $s_{2}=0$. However, it is known to be sharp since the solution map $u_{0} \mapsto u$ is not $\mathcal{C}^{3}$ in $H^{s}(\mathbb{R})$ as soon as $s<1 / 2$ (see [13]).

In the case $k=3$, the local well-posedness is known in $H^{s}(\mathbb{R}), s>1 / 3$ for small initial data [13] but only in $H^{s}(\mathbb{R}), s>3 / 4$ for large initial data. In 19 , we showed that (gBO) is $\mathcal{C}^{4}$-ill-posed in $H^{s}(\mathbb{R}), s<1 / 3$, in the sense that the flow-map $u_{0} \mapsto u$ fails to be $\mathcal{C}^{4}$. We prove here that well-posedness occurs in $H^{s}(\mathbb{R}), s>1 / 3$, and without smallness assumption on the initial data.

Concerning the case $k \geq 4$, global well-posedness in $H^{s}(\mathbb{R}), s>s_{k}$ was derived for small initial data by Molinet and Ribaud in [13. Later, by means of a gauge transformation, the same authors 12 removed the size restriction on the data and showed well-posedness in $H^{1 / 2}(\mathbb{R})$, whatever the value of $k$. By a refinement of their method, we reached in [19] the well-posedness in $H^{s}(\mathbb{R}), s>s_{k}$, but for high nonlinearities only ( $k \geq 12$ in fact). On the other hand, in the particular case $k=4$, Burq and Planchon [3] proved the local well-posedness in the critical space $\dot{H}^{1 / 4}(\mathbb{R})$. Inspired by their works, we extend in this paper the well-posedness to $\dot{H}^{s_{k}}(\mathbb{R})$ for any $k \geq 4$, and our method is flexible enough to get the result in the non-homogeneous space $H^{s_{k}}(\mathbb{R})$. A standard fixed point argument allows us to construct a unique solution in a subspace of $\dot{H}^{s_{k}}(\mathbb{R})$ with a continuous flow-map $u_{0} \mapsto u$. Recall that Biagioni and Linares [2] proved using solitary waves, that this map cannot be uniformly continuous in $\dot{H}^{s_{k}}(\mathbb{R})$. In the surcritical case $s<s_{k}$, we also know that the solution-map (if it exists) fails to be $\mathcal{C}^{k+1}$ in $H^{s}(\mathbb{R})$, see $[13$.

\section{Notations and main results}

\section{$2.1 \quad$ Notations}

For $A$ and $B$ two positive numbers, we write $A \lesssim B$ if it exists $c>0$ such that $A \leq c B$. Similarly define $A \gtrsim B, A \sim B$ if $A \geq c B$ and $A \lesssim B \lesssim A$ respectively. When the constant $c$ is large enough, we write $A \ll B$. For any $f \in \mathcal{S}^{\prime}(\mathbb{R})$, we use $\mathcal{F} f$ or $\hat{f}$ to denote its Fourier transform. For $1 \leq p \leq \infty$, $L^{p}$ is the standard Lebesgue space and its space-time versions $L_{x}^{p} L_{T}^{q}$ and $L_{T}^{q} L_{x}^{p}(T>0)$ are endowed with the norms

$$
\|f\|_{L_{x}^{p} L_{T}^{q}}=\|\| f\left\|_{L_{t}^{q}([-T ; T])}\right\|_{L_{x}^{p}(\mathbb{R})} \text { and }\|f\|_{L_{T}^{q} L_{x}^{p}}=\|\| f\left\|_{L_{x}^{p}(\mathbb{R})}\right\|_{L_{t}^{q}([-T ; T])} .
$$


The pseudo-differential operator $D_{x}^{\alpha}$ is defined by its Fourier symbol $|\xi|^{\alpha}$. We will denote by $P_{+}$and $P_{-}$the projection on respectively the positive and the negative spatial Fourier modes. Thus one has

$$
i \mathcal{H}=P_{+}-P_{-} .
$$

Let $\eta \in \mathcal{C}_{0}^{\infty}(\mathbb{R}), \eta \geq 0$, supp $\eta \subset\{1 / 2 \leq|\xi| \leq 2\}$ with $\sum_{-\infty}^{\infty} \eta\left(2^{-j} \xi\right)=1$ for $\xi \neq 0$. We set $p(\xi)=\sum_{j<-3} \eta\left(2^{-j} \xi\right)$ and consider, for all $j \in \mathbb{Z}$, the operator $Q_{j}$ defined by

$$
Q_{j}(f)=\mathcal{F}^{-1}\left(\eta\left(2^{-j} \xi\right) \hat{f}(\xi)\right) .
$$

We adopt the following summation convention. Any summation of the form $r \lesssim j, r \gg j, \ldots$ is a sum over the $r \in \mathbb{Z}$ such that $2^{r} \lesssim 2^{j} \ldots$, thus for instance $\sum_{r \lesssim j}=\sum_{r: 2^{r} \lesssim_{2}^{j}}$. We define then the operators $Q_{\lesssim j}=\sum_{r \lesssim j} Q_{r}$, $Q_{\ll j}=\sum_{r \ll j} Q_{r}$, etc. For $1 \leq p, q, r \leq \infty$ and $s \in \mathbb{R}$, let $\dot{\mathcal{B}}_{p}^{s, r}\left(L_{T}^{q}\right)$ be the homogeneous Besov space equipped with the norm

$$
\|f\|_{\dot{\mathcal{B}}_{p}^{s, r}\left(L_{T}^{q}\right)}=\left(\sum_{j \in \mathbb{Z}}\left[2^{j s}\left\|Q_{j} f\right\|_{L_{x}^{p} L_{T}^{q}}\right]^{r}\right)^{1 / r} .
$$

Finally for $s \in \mathbb{R}$ and $\theta \in[0,1]$, we define the solution space $\dot{\mathcal{S}}^{s, \theta}$ (where lives our solution $u$ ) and the nonlinear space $\dot{\mathcal{N}}^{s, \theta}$ (where lives the nonlinear term $\left.u^{k} \partial_{x} u\right)$ by

$$
\dot{\mathcal{S}}^{s, \theta}=\dot{\mathcal{B}}_{\frac{4}{1-\theta}}^{s+\frac{3 \theta-1}{4}, 2}\left(L_{T}^{\frac{2}{\theta}}\right), \quad \dot{\mathcal{N}}^{s, \theta}=\dot{\mathcal{B}}_{\frac{4}{3+\theta}}^{s+\frac{1-3 \theta}{4}}, 2\left(L_{T}^{\frac{2}{2-\theta}}\right) .
$$

\subsection{Main results}

We first state our well-posedness results in the case $k \geq 4$.

Theorem 2.1. Let $k \geq 4$ and $u_{0} \in \dot{H}^{s_{k}}(\mathbb{R})$. There exists $T=T\left(u_{0}\right)>0$ and a unique solution $u$ of $g B O$ such that $u \in \dot{Z}_{T}$ with

$$
\dot{Z}_{T}=\mathcal{C}\left([-T,+T], \dot{H}^{s_{k}}(\mathbb{R})\right) \cap \dot{X}^{s_{k}} \cap L_{x}^{k} L_{T}^{\infty} .
$$

Moreover, the flow map $u_{0} \mapsto u$ is locally Lipschitz from $\dot{H}^{s_{k}}(\mathbb{R})$ to $\dot{Z}_{T}$.

In the non-homogeneous case, one has the following result. 
Theorem 2.2. Let $k \geq 4$ and $u_{0} \in H^{s}(\mathbb{R}), s \geq s_{k}$. There exists $T=$ $T\left(u_{0}\right)>0$ and a unique solution $u$ of $g B O$ such that $u \in Z_{T}$ with

$$
Z_{T}=\mathcal{C}\left([-T,+T], H^{s}(\mathbb{R})\right) \cap X^{s} \cap L_{x}^{k} L_{T}^{\infty} .
$$

Moreover, the flow map $u_{0} \mapsto u$ is locally Lipschitz from $H^{s}(\mathbb{R})$ to $Z_{T}$.

Remark 2.1. We only obtain the Lipschitz continuity of the map $u_{0} \mapsto u$ in Theorems 2.1 and 2.2 in $\dot{H}^{s_{k}}(\mathbb{R})$ (resp. $\left.H^{s}(\mathbb{R})\right)$. As noticed in the introduction, the solution map given by Theorem 2.1 is not uniformly continuous from $\dot{H}^{s_{k}}(\mathbb{R})$ to $\mathcal{C}\left([-T, T], \dot{H}^{s_{k}}(\mathbb{R})\right)$. Moreover, when $s<s_{k}$, the flow map in Theorem 2.2 is no longer of class $\mathcal{C}^{k+1}$ in $H^{s}(\mathbb{R})$. It is not clear wether the map given by Theorems 2.1 and 2.2 is $\mathcal{C}^{k+1}$ or not.

Remark 2.2. The spaces $\dot{X}^{s_{k}}$ and $X^{s}$ will be defined in Section 3 and are directly related with the linear estimates for the linear Benjamin-Ono equation.

The main tools to prove Theorems 2.1 and 2.2 are the sharp Kato smoothing effect and the maximal in time inequality for the free solution $V(t) u_{0}$ where $V(t)=e^{i t \mathcal{H} \partial_{x}^{2}}$. Recall that for regular solutions, (gBO) is equivalent to its integral formulation

$$
u(t)=V(t) u_{0} \mp \int_{0}^{t} V\left(t-t^{\prime}\right)\left(u^{k}\left(t^{\prime}\right) \partial_{x} u\left(t^{\prime}\right)\right) d t^{\prime} .
$$

It is worth noticing that gBO provides a perfect balance between the derivative nonlinear term on one hand, and the available linear estimates on the other hand. Heuristically, one may use (2.1) to write

$$
\begin{aligned}
\left\|D_{x}^{s_{k}+1 / 2} u\right\|_{L_{x}^{\infty} L_{T}^{2}}+\|u\|_{L_{x}^{k} L_{T}^{\infty}} & \lesssim\left\|u_{0}\right\|_{\dot{H}^{s_{k}}}+\left\|D_{x}^{s_{k}-1 / 2} \partial_{x}\left(u^{k+1}\right)\right\|_{L_{x}^{1} L_{T}^{2}} \\
& \lesssim\left\|u_{0}\right\|_{\dot{H}^{s_{k}}}+\left\|D_{x}^{s_{k}+1 / 2} u\right\|_{L_{x}^{\infty} L_{T}^{2}}\|u\|_{L_{x}^{k} L_{T}^{\infty}}^{k}
\end{aligned}
$$

and perform a fixed point procedure. Unfortunately, such an argument fails for several reasons:

- First, it is not clear wether the second inequality holds true or not. Indeed, we used the fractional Leibniz rule (see the Appendix in [9], 12]) at the end points $L^{p}, p=1, \infty$. However, this inequality becomes true if one works in the associated Besov spaces $\dot{\mathcal{B}}_{\infty}^{s_{k}+1 / 2,2}\left(L_{T}^{2}\right) \cap \dot{\mathcal{B}}_{k}^{0,2}\left(L_{T}^{\infty}\right)$ and provides sharp well-posedness for small initial data, see [13]. 
- The term $\left\|V(t) u_{0}\right\|_{L_{x}^{k} L_{T}^{\infty}}$ will be small only if $\left\|u_{0}\right\|_{\dot{H}^{s_{k}}}$ is small as well, even for small $T$. Nevertheless, as noticed in [3], if we consider instead the difference $V(t) u_{0}-u_{0}$, then its $L_{x}^{k} L_{T}^{\infty}$-norm is small provided we restrict ourselves to a small interval $[-T, T]$ (see Lemma 3.5).

- We also need to get a better share of the derivative in the nonlinear term. By a standard paraproduct decomposition, we see that the worst contribution in $\partial_{x} u^{k+1}$ is given by $\pi(u, u)$ where

$$
\pi(f, g)=\sum_{j} \partial_{x} Q_{j}\left(\left(Q_{\ll j} f\right)^{k} Q_{\sim j} g\right) .
$$

The main idea is then to inject this term (or more precisely $\pi\left(V(t) u_{0}, u\right)$ ) in the linear part of the equation to get the variable-coefficient Schrödinger equation

$$
\partial_{t} u+\mathcal{H} \partial_{x}^{2} u+\pi\left(V(t) u_{0}, u\right)=f
$$

where $f$ will be a well-behaved term. Linear estimates for equation (2.2) are obtained by the localized gauge transform

$$
w_{j}=e^{\frac{i}{2} \int_{-\infty}^{x}\left(Q_{\ll j} u_{0}\right)^{k}} P_{+} Q_{j} u, \quad j \in \mathbb{Z} .
$$

Now we turn to the case $k=3$. By similar considerations, we obtain the following result.

Theorem 2.3. Let $k=3$ and $u_{0} \in H^{s}(\mathbb{R}), s>1 / 3$. There exists $T=$ $T\left(u_{0}\right)>0$ and a unique solution $u$ of g $g B O$ such that $u \in Z_{T}$ with

$$
Z_{T}=\mathcal{C}\left([-T,+T], H^{s}(\mathbb{R})\right) \cap X^{s} \cap L_{x}^{3} L_{T}^{\infty} .
$$

Moreover, the flow map $u_{0} \mapsto u$ is locally Lipschitz from $H^{s}(\mathbb{R})$ to $Z_{T}$.

This paper is organized as follows. In Section 3, we recall some sharp estimates related with the linear operator $V(t)$, and we derive linear estimates for equation (2.2). Section 4 is devoted to the case $k \geq 4$. Finally, we prove Theorem 2.3 in Section 5 .

\section{Linear estimates}

\subsection{Estimates for the linear $\mathrm{BO}$ equation}

This section deals with the well-known linear estimates for the BenjaminOno equation. Note that all results stated here hold as well for the Schrödinger operator $S(t)=e^{i t \partial_{x}^{2}}$. 
The following lemma summarizes the main estimates related to the group $V(t)$. See for instance [7, 8] for the proof.

Lemma 3.1. Let $\varphi \in \mathcal{S}(\mathbb{R})$, then

$$
\begin{aligned}
\|V(t) \varphi\|_{L_{T}^{\infty} L_{x}^{2}} & \lesssim\|\varphi\|_{L^{2}}, \\
\left\|D_{x}^{1 / 2} V(t) \varphi\right\|_{L_{x}^{\infty} L_{T}^{2}} & \lesssim\|\varphi\|_{L^{2}}, \\
\left\|D_{x}^{-1 / 4} V(t) \varphi\right\|_{L_{x}^{4} L_{T}^{\infty}} & \lesssim\|\varphi\|_{L^{2}} .
\end{aligned}
$$

Moreover, if $T \leq 1$ and $j \geq 0$,

$$
\begin{aligned}
\left\|Q_{\leq 0} V(t) \varphi\right\|_{L_{x}^{2} L_{T}^{\infty}} & \lesssim\left\|Q_{\leq 0} \varphi\right\|_{L^{2}} \\
2^{-j / 2}\left\|Q_{j} V(t) \varphi\right\|_{L_{x}^{2} L_{T}^{\infty}} & \lesssim\left\|Q_{j} \varphi\right\|_{L^{2}}
\end{aligned}
$$

Definition 3.1. A triplet $(\alpha, p, q) \in \mathbb{R} \times[2, \infty]^{2}$ is said to be 1-admissible if $(\alpha, p, q)=(1 / 2, \infty, 2)$ or

$$
4 \leq p<\infty, \quad 2<q \leq \infty, \quad \frac{2}{p}+\frac{1}{q} \leq \frac{1}{2}, \quad \alpha=\frac{1}{p}+\frac{2}{q}-\frac{1}{2} .
$$

By Sobolev embedding and interpolation between estimates (3.2) and (3.3) we obtain the following result.

Proposition 3.1 ([12]). If $(\alpha, p, q)$ is 1-admissible, then for all $\varphi$ in $\mathcal{S}(\mathbb{R})$,

$$
\left\|D_{x}^{\alpha} V(t) \varphi\right\|_{L_{x}^{p} L_{T}^{q}} \lesssim\|\varphi\|_{L^{2}} .
$$

Now we define our resolution spaces.

Definition 3.2. Let $k \geq 4$ and $s \in \mathbb{R}$ be fixed. For $0<\varepsilon \ll 1$, we define the spaces $\dot{X}^{s}=\dot{\mathcal{S}}^{s, \varepsilon} \cap \dot{\mathcal{S}}^{s, 1}$ endowed with the norm

$$
\|u\|_{\dot{X}^{s}}=\|u\|_{\dot{\mathcal{S}}^{s, \varepsilon}}+\|u\|_{\dot{\mathcal{S}}^{s, 1}} .
$$

At this stage it is important to remark that $\dot{X}^{s}$ does not contain any $L_{T}^{\infty}$ component. As a consequence, for each $u \in \dot{X}^{s}$ and $\eta>0$ fixed, we can choose $T=T(u)$ such that $\|u\|_{\dot{X}^{s}}<\eta$.

In the case $k=3$, we shall require the following result which is not covered by Proposition 3.1

Lemma $3.2([12])$. Let $0<T \leq 1$ and $s>1 / 3$. Then it holds that

$$
\|V(t) \varphi\|_{L_{x}^{3} L_{T}^{\infty}} \lesssim\|\varphi\|_{H^{s}}, \quad \forall \varphi \in \mathcal{S}(\mathbb{R}) .
$$


We next state the $L_{x}^{p} L_{T}^{q}$ and $L_{T}^{q} L_{x}^{p}$ estimates for the linear operator $f \mapsto \int_{0}^{t} V\left(t-t^{\prime}\right) f\left(t^{\prime}\right) d t^{\prime}$.

Lemma 3.3 ([12]). Let $\alpha \in \mathbb{R}$, and $2<p, q \leq \infty$ such that for all $\varphi \in \mathcal{S}(\mathbb{R})$,

$$
\left\|D_{x}^{\alpha} V(t) \varphi\right\|_{L_{x}^{p} L_{T}^{q}} \lesssim\|\varphi\|_{L^{2}} .
$$

Then for all $f \in \mathcal{S}\left(\mathbb{R}^{2}\right)$,

$$
\begin{gathered}
\left\|D_{x}^{1 / 2} \int_{0}^{t} V\left(t-t^{\prime}\right) f\left(t^{\prime}\right) d t^{\prime}\right\|_{L_{T}^{\infty} L_{x}^{2}} \lesssim\|f\|_{L_{x}^{1} L_{T}^{2}}, \\
\left\|D_{x}^{\alpha+1 / 2} \int_{0}^{t} V\left(t-t^{\prime}\right) f\left(t^{\prime}\right) d t^{\prime}\right\|_{L_{x}^{p} L_{T}^{q}} \lesssim\|f\|_{L_{x}^{1} L_{T}^{2}} .
\end{gathered}
$$

Similarly, if

$$
\left\|D_{x}^{\alpha} V(t) \varphi\right\|_{L_{x}^{p} L_{T}^{q}} \lesssim\|\varphi\|_{H^{s}}
$$

for any $\varphi \in \mathcal{S}(\mathbb{R})$, then

$$
\left\|D_{x}^{\alpha+1 / 2} \int_{0}^{t} V\left(t-t^{\prime}\right) f\left(t^{\prime}\right) d t^{\prime}\right\|_{L_{x}^{p} L_{T}^{q}} \lesssim\left\|\left\langle D_{x}\right\rangle^{s} f\right\|_{L_{x}^{1} L_{T}^{2}}
$$

We shall need the following Besov version of Lemma 3.3.

Lemma 3.4. Let $k \geq 4$. For all $f \in \mathcal{S}(\mathbb{R})^{2}$,

$$
\left\|\int_{0}^{t} V\left(t-t^{\prime}\right) f\left(t^{\prime}\right) d t^{\prime}\right\|_{L_{x}^{k} L_{T}^{\infty}} \lesssim\|f\|_{\dot{\mathcal{N}}^{s}, 1} .
$$

Proof. Note that the triplets $(1 / 2, \infty, 2)$ and $\left(-s_{k}, k, \infty\right)$ are both 1 -admissible. In particular we deduce

$$
\left\|\int_{-T}^{T} D_{x}^{1 / 2} V\left(-t^{\prime}\right) h\left(t^{\prime}\right) d t^{\prime}\right\|_{L^{2}} \lesssim\|h\|_{L_{x}^{1} L_{T}^{2}}, \quad \forall h \in \mathcal{S}\left(\mathbb{R}^{2}\right),
$$

which is the dual estimate of (3.7) for $(\alpha, p, q)=(1 / 2, \infty, 2)$. Since $L^{2}=$ $\dot{\mathcal{B}}_{2}^{0,2}$, we infer

$$
\left\|\int_{-T}^{T} D_{x}^{1 / 2} V\left(-t^{\prime}\right) h\left(t^{\prime}\right) d t^{\prime}\right\|_{L^{2}} \lesssim\|h\|_{\dot{\mathcal{B}}_{1}^{0,2}\left(L_{T}^{2}\right)}, \quad \forall h \in \mathcal{S}\left(\mathbb{R}^{2}\right) .
$$

The usual $T T^{*}$ argument provides

$$
\left\|\int_{-T}^{T} V\left(t-t^{\prime}\right) f\left(t^{\prime}\right) d t^{\prime}\right\|_{L_{x}^{k} L_{T}^{\infty}} \lesssim\|f\|_{\dot{\mathcal{B}}_{1}^{-1 / k, 2}\left(L_{T}^{2}\right)} .
$$

We can conclude with the Christ-Kiselev lemma for reversed norms (Theorem B in [3]). 


\subsection{Linear estimates for equation (2.2)}

Here and hereafter we take $k \geq 4$, the special case $k=3$ will be discussed in Section 5 .

Next lemma will be crucial in the proof of our main results.

Lemma 3.5. Let $k \geq 4$ and $u_{0} \in \dot{H}^{s_{k}}$. For any $\eta>0$, there exists $T=$ $T\left(u_{0}\right)$ such that

$$
\left\|V(t) u_{0}-u_{0}\right\|_{L_{x}^{k} L_{T}^{\infty}}<\eta .
$$

Proof. Let $N>0$ to be chosen later. One has

$$
\left\|V(t) u_{0}-u_{0}\right\|_{L_{x}^{k} L_{T}^{\infty}} \lesssim \sum_{|j|<N}\left\|Q_{j}\left(V(t) u_{0}-u_{0}\right)\right\|_{L_{x}^{k} L_{T}^{\infty}}+\left(\sum_{|j|>N}\left\|Q_{j} u_{0}\right\|_{\dot{H}^{s} k}^{2}\right)^{1 / 2} .
$$

Note that $v=V(t) u_{0}-u_{0}$ solves the equation

$$
\partial_{t} v+\mathcal{H} \partial_{x}^{2} v=-\mathcal{H} \partial_{x}^{2} u_{0}
$$

with zero initial data. Thus $V(t) u_{0}-u_{0}=\int_{0}^{t} V\left(t-t^{\prime}\right) \mathcal{H} \partial_{x}^{2} u_{0} d t^{\prime}$ and

$$
\begin{aligned}
\sum_{|j|<N}\left\|Q_{j}\left(V(t) u_{0}-u_{0}\right)\right\|_{L_{x}^{k} L_{T}^{\infty}} & \lesssim \sum_{|j|<N} 2^{2 j}\left\|\int_{0}^{t} V\left(t^{\prime}\right) Q_{j} u_{0} d t^{\prime}\right\|_{L_{x}^{k} L_{T}^{\infty}} \\
& \lesssim T \sum_{|j|<N} 2^{2 j}\left\|V(t) Q_{j} u_{0}\right\|_{L_{x}^{k} L_{T}^{\infty}} \\
& \lesssim T 2^{2 N}\left\|u_{0}\right\|_{\dot{H}^{s} k} .
\end{aligned}
$$

It suffices now to choose sufficiently large $N$ and then $T$ small enough.

Let us turn back to the nonlinear gBO equation. The sign of the nonlinearity is irrelevant in the study of the local problem, and we choose for convenience the plus sign.

Using standard paraproduct rearrangements, we can rewrite the nonlin- 
ear term in (gBO) as follows:

$$
\begin{aligned}
\partial_{x} Q_{j}\left(u^{k+1}\right) & =\partial_{x} Q_{j}\left(\lim _{r \rightarrow \infty}\left(Q_{<r} u\right)^{k+1}\right) \\
& =\partial_{x} Q_{j}\left(\sum_{-\infty}^{\infty}\left(Q_{<r+1} u\right)^{k+1}-\left(Q_{<r} u\right)^{k+1}\right) \\
& =\partial_{x} Q_{j}\left(\sum_{-\infty}^{\infty} Q_{r} u\left(Q_{\lesssim r} u\right)^{k}\right) \\
& =\partial_{x} Q_{j}\left(\sum_{r \sim j} Q_{r} u\left(Q_{\ll r} u\right)^{k}\right)+\partial_{x} Q_{j}\left(\sum_{r \gtrsim j}\left(Q_{\sim r} u\right)^{2}\left(Q_{{ }_{\supset}} u\right)^{k-1}\right) \\
& =\partial_{x} Q_{j}\left(\left(Q_{\ll j} u\right)^{k} Q_{\sim j} u\right)-g_{j} .
\end{aligned}
$$

We set

$$
\pi(f, g)=\sum_{j} \partial_{x} Q_{j}\left(\left(Q_{\ll j} f\right)^{k} Q_{\sim j} g\right)
$$

so that gBO reads

$$
\partial_{t} u+\mathcal{H} \partial_{x}^{2} u+\pi(u, u)=g(t, x)
$$

with

$$
g=\sum_{j} g_{j}
$$

Setting

$$
f=\pi\left(u_{L}, u\right)-\pi(u, u)+g
$$

where $u_{L}=V(t) u_{0}$ is the solution of the free $\mathrm{BO}$ equation, we see that (gBO) is equivalent to

$$
\partial_{t} u+\mathcal{H} \partial_{x}^{2} u+\pi\left(u_{L}, u\right)=f(t, x) .
$$

We intend to solve (gBO) by a fixed point procedure on the Duhamel formulation of (3.12):

$$
u(t)=U(t) u_{0}-\int_{0}^{t} U\left(t-t^{\prime}\right) f\left(t^{\prime}\right) d t^{\prime},
$$

where $U(t) \varphi$ is solution to

$$
\partial_{t} u+\mathcal{H} \partial_{x}^{2} u+\pi\left(V(t) u_{0}, u\right)=0, \quad u(0)=\varphi .
$$

It is worth noticing that $U(t)$ depends on the data $u_{0}$. 
Setting $u_{j}=Q_{j} u$ and $f_{j}=Q_{j} f$, we get from (3.12) that

$\partial_{t} u_{j}+\mathcal{H} \partial_{x}^{2} u_{j}+\partial_{x}\left(\left(u_{0, \ll j}\right)^{k} u_{j}\right)=\partial_{x}\left[\left(\left(u_{0, \ll j}\right)^{k}-\left(u_{L, \ll j}\right)^{k}\right) u_{j}\right]-\partial_{x}\left[Q_{j},\left(u_{L, \ll j}\right)^{k}\right] u_{\sim j}+f_{j}$

and we will denote by $R_{j}$ the right-hand side. Now take the positive frequencies and set $v_{j}=P_{+} u_{j}$ :

$$
i \partial_{t} v_{j}+\partial_{x}^{2} v_{j}+i \partial_{x}\left(\left(u_{0, \ll j}\right)^{k} v_{j}\right)=i P_{+} R_{j} .
$$

With $b_{\ll j}=\frac{1}{2}\left(u_{0, \ll j}\right)^{k}$, we obtain

$$
i \partial_{t} v_{j}+\left(\partial_{x}+i b_{\ll j}\right)^{2} v_{j}=g_{j}
$$

with

$$
g_{j}=-i \partial_{x} b_{\ll j} \cdot v_{j}-b_{\ll j}^{2} v_{j}+i P_{+} R_{j} .
$$

Lemma 3.6. Let $v_{j}$ be a solution to (3.13) with initial data $v_{0, j} \in \dot{H}^{s_{k}} \cap \dot{H}^{s}$. Then there exists $C=C\left(u_{0}\right)$ such that

$$
\left\|v_{j}\right\|_{\dot{X}^{s}} \leq C\left\|v_{0, j}\right\|_{\dot{H}^{s}}+C\left\|g_{j}\right\|_{\dot{\mathcal{N}}^{s, 1}} .
$$

Proof. We define $w_{j}$ by

$$
w_{j}=e^{i \int^{x} b_{\ll j}} v_{j}
$$

Then we easily check that $w_{j}$ solves

$$
i \partial_{t} w_{j}+\partial_{x}^{2} w_{j}=e^{i \int^{x} b_{\ll j}} g_{j}
$$

From the well-known linear estimates on the Schrödinger equation (Lemmas 3.1 3.3. we infer

$$
\left\|\partial_{x} w_{j}\right\|_{L_{x}^{\infty} L_{T}^{2}} \lesssim\left\|e^{i \int^{x} b_{\ll j}} v_{0, j}\right\|_{\dot{H}^{1 / 2}}+\left\|g_{j}\right\|_{L_{x}^{1} L_{T}^{2}}
$$

Since $\partial_{x} w_{j}=e^{i \int^{x} b_{\ll j}}\left(\partial_{x} v_{j}+b_{\ll j} v_{j}\right)$, we have

$$
\begin{aligned}
\left\|\partial_{x} v_{j}\right\|_{L_{x}^{\infty} L_{T}^{2}} & \lesssim\left\|\partial_{x} w_{j}\right\|_{L_{x}^{\infty} L_{T}^{2}}+\left\|b_{\ll j} v_{j}\right\|_{L_{x}^{\infty} L_{T}^{2}} \\
& \lesssim\left\|\partial_{x} w_{j}\right\|_{L_{x}^{\infty} L_{T}^{2}}+2^{-j}\left\|b_{\ll j}\right\|_{L^{\infty}}\left\|\partial_{x} v_{j}\right\|_{L_{x}^{\infty} L_{T}^{2}}
\end{aligned}
$$

On the other hand, we can make $2^{-j}\left\|\left(u_{0, \ll j}\right)^{k}\right\|_{L^{\infty}}$ as small as desired by choosing the implicit constant $J=J\left(u_{0}\right)$ in $u_{0, \ll j}$ large enough:

$$
2^{-j}\left\|\left(u_{0,<j-J}\right)^{k}\right\|_{L^{\infty}} \lesssim 2^{-j} 2^{j-J}\left\|u_{0}\right\|_{L^{k}}^{k} \lesssim c\left(u_{0}\right) 2^{-J} \ll 1
$$


It follows that

$$
\left\|\partial_{x} v_{j}\right\|_{L_{x}^{\infty} L_{T}^{2}} \lesssim\left\|e^{i \int^{x} b_{\ll j}} v_{0, j}\right\|_{\dot{H}^{1 / 2}}+\left\|g_{j}\right\|_{L_{x}^{1} L_{T}^{2}} .
$$

We now use the fractional Leibniz rule (Theorem A.12 in [9]) and Bernstein inequality to estimate the first term in the right-hand side,

$$
\begin{aligned}
\left\|e^{i \int^{x} b_{\ll j}} v_{0, j}\right\|_{\dot{H}^{1 / 2}} & \lesssim\left\|e^{i \int^{x} b_{\ll j}}\right\|_{L^{\infty}}\left\|v_{0, j}\right\|_{\dot{H}^{1 / 2}}+\| D_{x}^{1 / 2} e^{i \int^{x} b_{\ll j}\left\|_{L^{\infty}}\right\| v_{0, j} \|_{L^{2}}} \\
& \lesssim\left\|v_{0, j}\right\|_{\dot{H}^{1 / 2}}+\left\|\left(u_{0, \ll j}\right)^{k}\right\|_{L^{2}}\left\|v_{0, j}\right\|_{L^{2}} \\
& \lesssim\left(1+\left\|u_{0}\right\|_{L^{k}}^{k}\right)\left\|v_{0, j}\right\|_{\dot{H}^{1 / 2}}
\end{aligned}
$$

Since $v_{j}, g_{j}$ as well as $v_{0, j}$ are frequency localized, we conclude

$$
\left\|v_{j}\right\|_{\dot{\mathcal{B}}_{\infty}^{s+1 / 2,2}\left(L_{T}^{2}\right)} \lesssim\left\|v_{0, j}\right\|_{\dot{H}^{s}}+\left\|g_{j}\right\|_{\dot{\mathcal{B}}_{1}^{s-1 / 2,2}\left(L_{T}^{2}\right)} .
$$

We also need $L_{x}^{4} L_{T}^{\infty}$-norm estimates. Our equation can be rewritten as

$$
i \partial_{t} v_{j}+\partial_{x}^{2} v_{j}=g_{j}+h_{j}
$$

with

$$
h_{j}=b_{\ll j}^{2} v_{j}-i \partial_{x}\left(b_{\ll j} v_{j}\right)-i b_{\ll j} \partial_{x} v_{j} .
$$

Thus we get from Lemmas $3.1+3.3$ that

$$
\left\|v_{j}\right\|_{\dot{\mathcal{B}}_{4}^{s-1 / 4,2}\left(L_{T}^{\infty}\right)} \lesssim\left\|v_{0, j}\right\|_{\dot{H}^{s}}+\left\|g_{j}\right\|_{\dot{\mathcal{B}}_{1}^{s-1 / 2,2}\left(L_{T}^{2}\right)}+\left\|h_{j}\right\|_{\dot{\mathcal{B}}_{1}^{s-1 / 2,2}\left(L_{T}^{2}\right)} .
$$

We bound the $h_{j}$ contribution with (3.15):

$$
\begin{aligned}
\left\|b_{\ll j}^{2} v_{j}\right\|_{\dot{\mathcal{B}}_{1}^{s-1 / 2,2}\left(L_{T}^{2}\right)} & \lesssim 2^{j(s-1 / 2)}\left\|b_{\ll j}^{2}\right\|_{L^{1}}\left\|v_{j}\right\|_{L_{x}^{\infty} L_{T}^{2}} \\
& \lesssim\left(2^{-j / 2}\left\|b_{\ll j}\right\|_{L^{2}}\right)^{2}\left\|v_{j}\right\|_{\dot{\mathcal{B}}_{1}^{s+1 / 2,2}\left(L_{T}^{2}\right)} \\
& \lesssim\|b\|_{L^{1}}^{2}\left(\left\|v_{0, j}\right\|_{\dot{H}^{s}}+\left\|g_{j}\right\|_{\dot{\mathcal{B}}_{1}^{s-1 / 2,2}\left(L_{T}^{2}\right)}\right),
\end{aligned}
$$

and

$$
\begin{aligned}
\left\|\partial_{x}\left(b_{\ll j} v_{j}\right)+b_{\ll j} \partial_{x} v_{j}\right\|_{\dot{\mathcal{B}}_{1}^{s-1 / 2,2}\left(L_{T}^{2}\right)} & \lesssim 2^{j(s+1 / 2)}\left\|b_{\ll j}\right\|_{L^{1}}\left\|v_{j}\right\|_{L_{x}^{\infty} L_{T}^{2}} \\
& \lesssim\|b\|_{L^{1}}\left(\left\|v_{0, j}\right\|_{\dot{H}^{s}}+\left\|g_{j}\right\|_{\dot{\mathcal{B}}_{1}^{s-1 / 2,2}\left(L_{T}^{2}\right)}\right) .
\end{aligned}
$$

Therefore,

$$
\left\|v_{j}\right\|_{\dot{\mathcal{B}}_{4}^{s-1 / 4,2}\left(L_{T}^{\infty}\right)} \lesssim\left\|v_{0, j}\right\|_{\dot{H}^{s}}+\left\|g_{j}\right\|_{\dot{\mathcal{B}}_{1}^{s-1 / 2,2}\left(L_{T}^{2}\right)}
$$

and the claim follows by interpolation between (3.16) and (3.15). 
We are now ready to prove the main linear estimate on equation (3.12).

Proposition 3.2. Let $u$ be a solution of (3.12) with initial data $u_{0} \in \dot{H}^{s} \cap$ $\dot{H}^{s_{k}}, s \in \mathbb{R}$. Then there exists $T=T\left(u_{0}\right)>0$ and $C=C\left(u_{0}\right)$ such that on $[-T,+T]$

$$
\|u\|_{\dot{X}^{s}} \leq C\left\|u_{0}\right\|_{\dot{H}^{s}}+C\|f\|_{\dot{\mathcal{N}}^{s, 1}} \text {. }
$$

Proof. Using that $\left|P_{+} u_{j}\right|=\left|P_{-} u_{j}\right|$ (since $u$ is real) and Lemma 3.6, we infer

$$
\begin{aligned}
\left\|u_{j}\right\|_{\dot{X}^{s}} \lesssim\left\|v_{j}\right\|_{\dot{X}^{s}} \lesssim & \left\|Q_{j} u_{0}\right\|_{\dot{X}^{s}}+\left\|f_{j}\right\|_{\dot{\mathcal{N}}^{s, 1}}+\left\|\partial_{x}\left(u_{0, \ll j}\right)^{k} v_{j}\right\|_{\dot{\mathcal{N}}^{s, 1}}+\left\|\left(u_{0, \ll j}\right)^{2 k} v_{j}\right\|_{\dot{\mathcal{N}}^{s, 1}} \\
& +\left\|\partial_{x}\left[\left(\left(u_{0, \ll j}\right)^{k}-\left(u_{L, \ll j}\right)^{k}\right) u_{j}\right]\right\|_{\dot{\mathcal{N}}^{s, 1}}+\left\|\partial_{x}\left[Q_{j},\left(u_{L, \ll j}\right)^{k}\right] u_{\sim j}\right\|_{\dot{\mathcal{N}}^{s, 1}} \\
= & \left\|Q_{j} u_{0}\right\|_{\dot{X}^{s}}+\left\|f_{j}\right\|_{\dot{\mathcal{N}}^{s, 1}}+A+B+C+D
\end{aligned}
$$

We bound $A$ by

$$
\begin{aligned}
A & \lesssim 2^{j(s-1 / 2)}\left\|\partial_{x}\left(u_{0, \ll j}\right)^{k} v_{j}\right\|_{L_{x}^{1} L_{T}^{2}} \lesssim 2^{-j}\left\|\partial_{x}\left(u_{0, \ll j}\right)^{k}\right\|_{L^{1}} 2^{j(s+1 / 2)}\left\|v_{j}\right\|_{L_{x}^{\infty} L_{T}^{2}} \\
& \lesssim 2^{-j}\left\|\partial_{x}\left(u_{0, \ll j}\right)^{k}\right\|_{L^{1}}\left\|v_{j}\right\|_{\dot{X}^{s}} .
\end{aligned}
$$

As previously, $2^{-j}\left\|\partial_{x}\left(u_{0, \ll j}\right)^{k}\right\|_{L^{1}}$ can be made as small as needed by choosing the implicit constant $J=J\left(u_{0}\right)$ in $u_{0, \ll j}$ large enough:

$$
2^{-j}\left\|\partial_{x}\left(u_{0,<j-J}\right)^{k}\right\|_{L^{1}} \lesssim 2^{-j} 2^{j-J}\left\|u_{0}\right\|_{L^{k}}^{k} \lesssim c\left(u_{0}\right) 2^{-J} \ll 1 .
$$

One proceeds similarly for $B$ :

$$
\begin{aligned}
B & \lesssim 2^{j(s-1 / 2)}\left\|\left(u_{0, \ll j}\right)^{2 k} v_{j}\right\|_{L_{x}^{1} L_{T}^{2}} \\
& \lesssim 2^{-j}\left\|\left(u_{0, \ll j}\right)^{k}\right\|_{L^{\infty}}\left\|u_{0}\right\|_{L^{k}}^{k} 2^{j(s+1 / 2)}\left\|v_{j}\right\|_{L_{x}^{\infty} L_{T}^{2}} \\
& \ll\left\|v_{j}\right\|_{\dot{X}^{s}} .
\end{aligned}
$$

Now we estimate $C$ :

$$
\begin{aligned}
C & \lesssim 2^{j(s-1 / 2)}\left\|\partial_{x}\left[\left(\left(u_{0, \ll j}\right)^{k}-\left(u_{L, \ll j}\right)^{k}\right) u_{j}\right]\right\|_{L_{x}^{1} L_{T}^{2}} \\
& \lesssim 2^{j(s+1 / 2)}\left\|\left(u_{0, \ll j}\right)^{k}-\left(u_{L, \ll j}\right)^{k}\right\|_{L_{x}^{1} L_{T}^{2}}\left\|u_{j}\right\|_{L_{x}^{\infty} L_{T}^{2}} \\
& \lesssim\left\|u_{0}-u_{L}\right\|_{L_{x}^{k} L_{T}^{\infty}}\left(\left\|u_{0}\right\|_{L^{k}}^{k-1}+\left\|u_{L}\right\|_{L_{x}^{k} L_{T}^{\infty}}^{k-1}\right)\left\|u_{j}\right\|_{\dot{X}^{s}} \\
& \ll\left\|u_{j}\right\|_{\dot{X}^{s}}
\end{aligned}
$$


by Lemma 3.5. Finally we deal with term $D$. By commutator lemma (Lemma 2.4 in [3]), we get

$$
\begin{aligned}
& D \lesssim 2^{j(s-1 / 2)}\left\|\partial_{x}\left[Q_{j},\left(u_{L, \ll j}\right)^{k}\right] u_{j}\right\|_{L_{x}^{1} L_{T}^{2}} \\
& \lesssim 2^{j(s-1 / 2)} 2^{-j}\left\|\partial_{x}\left(u_{L, \ll j}\right)^{k}\right\|_{L_{x}^{4-\varepsilon}} L_{T}^{\frac{2}{\varepsilon}}\left\|\partial_{x} u_{j}\right\|_{L_{x}^{\frac{4}{\varepsilon}}} \frac{2}{L_{T}^{1-\varepsilon}} \\
& \lesssim 2^{-j} 2^{3 j \varepsilon / 4}\left\|\partial_{x}\left(u_{L, \ll j}\right)^{k}\right\|_{L_{x}^{4-\varepsilon}} L_{T}^{\frac{2}{\varepsilon}}\left\|u_{j}\right\|_{\dot{\mathcal{S}}^{s, 1-\varepsilon}}
\end{aligned}
$$

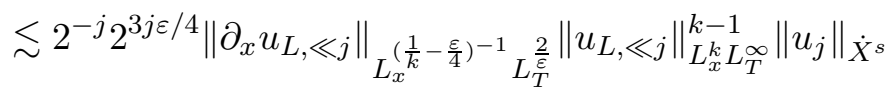

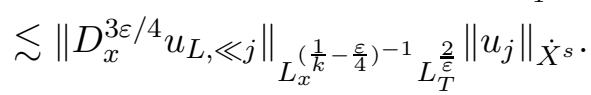

Since the triplet $\left(\frac{3 \varepsilon}{4}-s_{k},\left(\frac{1}{k}-\frac{\varepsilon}{4}\right)^{-1}, \frac{2}{\varepsilon}\right)$ is 1 -admissible, for any $\eta>0$, we can choose $T>0$ small enough such that

$$
\left\|D_{x}^{3 \varepsilon / 4} u_{L}\right\|_{L_{x}^{\left(\frac{1}{k}-\frac{\varepsilon}{4}\right)^{-1}} L_{T}^{\frac{2}{\varepsilon}}<\eta}
$$

Gathering all these estimates we infer

$$
\left\|u_{j}\right\|_{\dot{X}^{s}} \lesssim\left\|Q_{j} u_{0}\right\|_{\dot{X}^{s}}+\left\|f_{j}\right\|_{\dot{\mathcal{N}}^{s, 1}} .
$$

Summing this inequality over $j$ finishes the proof of Proposition 3.2

We also need $L_{x}^{k} L_{T}^{\infty}$-norm estimates.

Proposition 3.3. Let $u$ be a solution of (3.12) with initial data $u_{0} \in \dot{H}^{s_{k}}$. Then there exists $T>0$ and $C=C\left(u_{0}\right)$ such that

$$
\|u\|_{L_{x}^{k} L_{T}^{\infty}} \leq C\left\|u_{0}\right\|_{\dot{H}^{s} k}+C\|f\|_{\dot{\mathcal{N}}^{s_{k}}, 1}
$$

Moreover, if $u_{0} \in \dot{H}^{s} \cap \dot{H}^{s_{k}}, s \in \mathbb{R}$, then

$$
\|u\|_{L_{T}^{\infty} \dot{H}_{x}^{s}} \leq C\left\|u_{0}\right\|_{\dot{H}^{s}}+C\|f\|_{\dot{\mathcal{N}}^{s, 1}} .
$$

Proof. We can rewrite our equation as

$$
u=u_{L}-\int_{0}^{t} V\left(t-t^{\prime}\right)\left(f-\pi\left(u_{L}, u\right)\right) d t^{\prime} .
$$

By virtue of Lemma 3.4 and Lemma 3.3, we deduce

$$
\|u\|_{L_{x}^{k} L_{T}^{\infty}} \lesssim\left\|u_{0}\right\|_{\dot{H}^{s_{k}}}+\|f\|_{\dot{\mathcal{N}}^{s_{k}, 1}}+\left\|\pi\left(u_{L}, u\right)\right\|_{\dot{\mathcal{N}}^{s_{k}, 1}},
$$




$$
\|u\|_{L_{T}^{\infty} \dot{H}_{x}^{s}} \lesssim\left\|u_{0}\right\|_{\dot{H}^{s}}+\|f\|_{\dot{\mathcal{N}}^{s, 1}}+\left\|\pi\left(u_{L}, u\right)\right\|_{\dot{\mathcal{N}}^{s, 1}} .
$$

Then we get

$$
\begin{aligned}
\left\|\pi\left(u_{L}, u\right)\right\|_{\dot{\mathcal{N}}^{s, 1}} & \lesssim\left(\sum_{j}\left[2^{j(s+1 / 2)}\left\|\left(u_{L, \ll j}\right)^{k} u_{\sim j}\right\|_{L_{x}^{1} L_{T}^{2}}\right]^{2}\right)^{1 / 2} \\
& \lesssim\left\|u_{L}\right\|_{L_{x}^{k} L_{T}^{\infty}}^{k}\left(\sum_{j}\left[2^{j(s+1 / 2)}\left\|u_{\sim j}\right\|_{L_{x}^{\infty} L_{T}^{2}}\right]^{2}\right)^{1 / 2} \\
& \lesssim\left\|u_{0}\right\|_{\dot{H}^{s} k}^{k}\|u\|_{\dot{X}^{s}} \\
& \lesssim C\left(u_{0}\right)\left(\left\|u_{0}\right\|_{\dot{H}^{s}}+\|f\|_{\dot{\mathcal{N}}^{s, 1}}\right)
\end{aligned}
$$

by Proposition 3.2 .

\section{Well-posedness for $k \geq 4$}

\subsection{Nonlinear estimates}

Now we estimate the right-hand side of $(3.12)$ in $\dot{\mathcal{N}}^{s, 1}$-norm.

Proposition 4.1. For any $u \in \dot{X}^{s} \cap L_{x}^{k} L_{T}^{\infty}$, we have

$$
\left\|\pi\left(u_{L}, u\right)-\pi(u, u)\right\|_{\dot{\mathcal{N}}^{s, 1}} \lesssim\left\|u_{L}-u\right\|_{L_{x}^{k} L_{T}^{\infty}}\left(\left\|u_{L}\right\|_{L_{x}^{k} L_{T}^{\infty}}^{k-1}+\|u\|_{L_{x}^{k} L_{T}^{\infty}}^{k-1}\right)\|u\|_{\dot{X}^{s}}
$$

and

$$
\|g\|_{\dot{\mathcal{N}}^{s, 1}} \lesssim\|u\|_{L_{x}^{k} L_{T}^{\infty}}^{k-1}\|u\|_{\dot{X}^{s}}^{2} .
$$

Proof. Set $u_{j}=Q_{j} u, u_{\ll j}=Q_{\ll j} u$, etc. Then:

$$
\begin{aligned}
\left\|\pi\left(u_{L}, u\right)-\pi(u, u)\right\|_{\dot{\mathcal{N}}^{s, 1}} & \lesssim\left(\sum_{j}\left[2^{j(s-1 / 2)}\left\|\partial_{x}\left[\left(\left(u_{L, \ll j}\right)^{k}-\left(u_{\ll j}\right)^{k}\right) u_{\sim j}\right]\right\|_{L_{x}^{1} L_{T}^{2}}\right]^{2}\right)^{1 / 2} \\
& \lesssim\left(\sum_{j}\left[2^{j(s+1 / 2)}\left\|\left(u_{L, \ll j}\right)^{k}-\left(u_{\ll j}\right)^{k}\right\|_{L_{x}^{1} L_{T}^{\infty}}\left\|u_{\sim j}\right\|_{L_{x}^{\infty} L_{T}^{2}}\right]^{2}\right)^{1 / 2} \\
& \lesssim\left\|u_{L}-u\right\|_{L_{x}^{k} L_{T}^{\infty}}\left(\left\|u_{L}\right\|_{L_{x}^{k} L_{T}^{\infty}}^{k-1}+\|u\|_{L_{x}^{k} L_{T}^{\infty}}^{k-1}\right)\|u\|_{\dot{X}^{s}} .
\end{aligned}
$$


We bound the second term by

$$
\begin{aligned}
& \|g\|_{\dot{\mathcal{N} s, 1}} \lesssim\left(\sum_{j}\left[2^{j(s+1 / 2)} \sum_{r \gtrsim j}\left\|\left(u_{\sim r}\right)^{2}\left(u_{\lesssim r}\right)^{k-1}\right\|_{L_{x}^{1} L_{T}^{2}}\right]^{2}\right)^{1 / 2}
\end{aligned}
$$

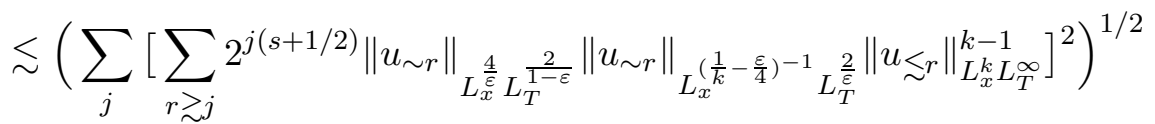

$$
\begin{aligned}
& \lesssim\|u\|_{L_{x}^{k} L_{T}^{\infty}}^{k-1} \sup _{r} 2^{3 \varepsilon r / 4}\left\|u_{\sim r}\right\|_{L_{x}^{\left(\frac{1}{k}-\frac{\varepsilon}{4}\right)^{-1}}{ }_{L_{T}^{\frac{2}{\varepsilon}}}} \\
& \times\left(\sum_{j}\left[\sum_{r \gtrsim j}\left(2^{(j-r)(s+1 / 2)}\right)\left(2^{r(s+1 / 2-3 \varepsilon / 4)}\left\|u_{\sim r}\right\|_{L_{x}^{\frac{4}{\varepsilon}} L_{T}^{1-\varepsilon}}\right)\right]^{2}\right)^{1 / 2} \\
& \lesssim\|u\|_{L_{x}^{k} L_{T}^{\infty}}^{k-1}\|u\|_{\dot{S}^{s, \varepsilon}}\left(\sum_{j \leq 0} 2^{j(s+1 / 2)}\right)\left(\sum_{j}\left[2^{j(s+1 / 2-3 \varepsilon / 4)}\left\|u_{\sim j}\right\|_{L_{x}^{\frac{4}{\varepsilon}} L_{T}^{1-\varepsilon}}\right]^{2}\right)^{1 / 2} \\
& \lesssim\|u\|_{L_{x}^{k} L_{T}^{\infty}}^{k-1}\|u\|_{\dot{X}^{s}}^{2}
\end{aligned}
$$

where we used discrete Young inequality.

\subsection{Existence in $\dot{H}^{s_{k}}(\mathbb{R})$}

Consider the map $F$ defined as

$$
F(u)=U(t) u_{0}-\int_{0}^{t} U\left(t-t^{\prime}\right) f\left(t^{\prime}\right) d t^{\prime} .
$$

We shall contract $F$ in the intersection of two balls:

$$
B_{M}\left(u_{0}, T\right)=\left\{u \in \dot{X}^{s_{k}} \cap L_{x}^{k} L_{T}^{\infty}:\left\|u-u_{0}\right\|_{L_{x}^{k} L_{T}^{\infty}} \leq \delta\right\}
$$

and

$$
B_{S}\left(u_{0}, T\right)=\left\{u \in \dot{X}^{s_{k}} \cap L_{x}^{k} L_{T}^{\infty}:\|u\|_{\dot{X}^{s} k} \leq \delta\right\}
$$

endowed with the norm

$$
\|u\|_{\dot{Y}_{T}}=\|u\|_{\dot{X}^{s} k}+\|u\|_{L_{x}^{k} L_{T}^{\infty}} .
$$

Gathering Propositions [3.2, 3.3 and 4.1 (with $s=s_{k}$ ) we find that there exists $C=C\left(u_{0}\right)>1$ such that

$$
\begin{aligned}
& \|F(u)\|_{\dot{X}^{s_{k}}} \leq C\left\|U(t) u_{0}\right\|_{\dot{X}^{s_{k}}}+C\left(1+\left\|u-u_{0}\right\|_{L_{x}^{k} L_{T}^{\infty}}^{k-1}\right)\|u\|_{\dot{X}^{s_{k}}}^{2} \\
& \quad+C\left(\left\|u_{L}-u_{0}\right\|_{L_{x}^{k} L_{T}^{\infty}}+\left\|u-u_{0}\right\|_{L_{x}^{k} L_{T}^{\infty}}\right)\left(1+\left\|u-u_{0}\right\|_{L_{x}^{k} L_{T}^{\infty}}^{k-1}\right)\|u\|_{\dot{X}^{s_{k}}}
\end{aligned}
$$


and

$$
\begin{aligned}
\| F(u) & -u_{0}\left\|_{L_{x}^{k} L_{T}^{\infty}} \leq\right\| U(t) u_{0}-u_{0}\left\|_{L_{x}^{k} L_{T}^{\infty}}+C\left(1+\left\|u-u_{0}\right\|_{L_{x}^{k} L_{T}^{\infty}}^{k-1}\right)\right\| u \|_{\dot{X}^{s} k}^{2} \\
& +C\left(\left\|u_{L}-u_{0}\right\|_{L_{x}^{k} L_{T}^{\infty}}+\left\|u-u_{0}\right\|_{L_{x}^{k} L_{T}^{\infty}}\right)\left(1+\left\|u-u_{0}\right\|_{L_{x}^{k} L_{T}^{\infty}}^{k-1}\right)\|u\|_{\dot{X}^{s_{k}}} .
\end{aligned}
$$

We can choose $T=T\left(u_{0}\right)$ small enough so that the quantities $\left\|U(t) u_{0}\right\|_{\dot{X}^{s_{k}}}$, $\left\|u_{L}-u_{0}\right\|_{L_{x}^{k} L_{T}^{\infty}}$ and $\left\|U(t) u_{0}-u_{0}\right\|_{L_{x}^{k} L_{T}^{\infty}}$ are smaller than $\varepsilon=\frac{1}{128 C^{2}}$. Thus if $u \in B_{M} \cap B_{S}$, then

$$
\|F(u)\|_{\dot{X}^{s_{k}}} \leq 4 C \varepsilon+4 C \delta^{2}
$$

and

$$
\left\|F(u)-u_{0}\right\|_{L_{x}^{k} L_{T}^{\infty}} \leq 4 C \varepsilon+4 C \delta^{2} .
$$

Now we take $\delta=\frac{1}{8 C}$ so that $F(u)$ belongs to $B_{M} \cap B_{S}$. In the same way, for any $u_{1}$ and $u_{2}$ in $B_{M} \cap B_{S}$, one has

$$
\begin{aligned}
\left\|F\left(u_{1}\right)-F\left(u_{2}\right)\right\|_{\dot{Y}_{T}} \lesssim & \left\|f\left(u_{1}\right)-f\left(u_{2}\right)\right\|_{\dot{\mathcal{N}}^{s_{k}}, 1} \\
\lesssim & \left\|u_{L}-u_{1}\right\|_{L_{x}^{k} L_{T}^{\infty}}\left(1+\left\|u_{1}\right\|_{L_{x}^{k} L_{T}^{\infty}}^{k-1}\right)\left\|u_{1}-u_{2}\right\|_{\dot{X}^{s_{k}}} \\
& +\left\|u_{2}\right\|_{\dot{X}^{s_{k}}}\left(\left\|u_{1}\right\|_{L_{x}^{k} L_{T}^{\infty}}^{k-1}+\left\|u_{2}\right\|_{L_{x}^{k} L_{T}^{\infty}}^{k-1}\right)\left\|u_{1}-u_{2}\right\|_{L_{x}^{k} L_{T}^{\infty}} \\
& +\left\|u_{1}\right\|_{\dot{X}^{s_{k}}}^{2}\left(\left\|u_{1}\right\|_{L_{x}^{k} L_{T}^{\infty}}^{k-2}+\left\|u_{2}\right\|_{L_{x}^{k} L_{T}^{\infty}}^{k-2}\right)\left\|u_{1}-u_{2}\right\|_{L_{x}^{k} L_{T}^{\infty}} \\
& +\left\|u_{2}\right\|_{L_{x}^{k} L_{T}^{\infty}}^{k-1}\left(\left\|u_{1}\right\|_{\dot{X}^{s_{k}}}+\left\|u_{2}\right\|_{\dot{X}^{s} k}\right)\left\|u_{1}-u_{2}\right\|_{\dot{X}^{s_{k}}} .
\end{aligned}
$$

Therefore,

$$
\left\|F\left(u_{1}\right)-F\left(u_{2}\right)\right\|_{\dot{Y}_{T}} \lesssim(\varepsilon+\delta)\left\|u_{1}-u_{2}\right\|_{\dot{Y}_{T}}
$$

and for $\varepsilon, \delta$ small enough, $F: B_{M} \cap B_{S} \rightarrow B_{M} \cap B_{S}$ is contractive. There exists a solution $u$ in $B_{M} \cap B_{S}$.

The next step is to show that $u \in \mathcal{C}\left([-T,+T], \dot{H}^{s_{k}}(\mathbb{R})\right)$. Using (3.17) and Proposition 4.1, we obtain that $u \in L_{T}^{\infty} \dot{H}_{x}^{s_{k}}$. For any $t_{1}, t_{2} \in[0, T]$ with $t_{1}<t_{2}$, writing $u(t)$ as

$$
u(t)=V\left(t-t_{1}\right) u\left(t_{1}\right)-\int_{t_{1}}^{t} V\left(t-t^{\prime}\right) \partial_{x} u^{k+1}\left(t^{\prime}\right) d t^{\prime},
$$


we get

$$
\begin{aligned}
\left\|u\left(t_{1}\right)-u\left(t_{2}\right)\right\|_{\dot{H}^{s_{k}}} & \lesssim \sup _{t \in\left[t_{1}, t_{2}\right]}\left\|u(t)-u\left(t_{1}\right)\right\|_{\dot{H}^{s_{k}}} \\
& \lesssim \sup _{t \in\left[t_{1}, t_{2}\right]}\left\|u\left(t_{1}\right)-V\left(t-t_{1}\right) u\left(t_{1}\right)\right\|_{\dot{H}^{s_{k}}} \\
& +\left\|\int_{t_{1}}^{t} V\left(t-t^{\prime}\right) \partial_{x} u^{k+1}\left(t^{\prime}\right) d t^{\prime}\right\|_{L^{\infty}\left(t_{1}, t_{2} ; \dot{H}^{s} s_{k}\right)} \\
& \rightarrow 0
\end{aligned}
$$

as $t_{1} \rightarrow t_{2}$.

Now consider $u_{0,1}, u_{0,2} \in \dot{H}^{s_{k}}$ two initial data, and $u_{1}, u_{2} \in \dot{Z}_{T}$ satisfying

$$
\begin{aligned}
& u_{1}(t)=U_{1}(t) u_{0,1}-\int_{0}^{t} U_{1}\left(t-t^{\prime}\right) f_{1}\left(u_{1}\right)\left(t^{\prime}\right) d t^{\prime}, \\
& u_{2}(t)=U_{2}(t) u_{0,2}-\int_{0}^{t} U_{2}\left(t-t^{\prime}\right) f_{2}\left(u_{2}\right)\left(t^{\prime}\right) d t^{\prime},
\end{aligned}
$$

where $U_{j}(t) \varphi$ is solution to

$$
\partial_{t} u+\mathcal{H} \partial_{x}^{2} u+\pi\left(V(t) u_{0, j}, u\right)=0, \quad u(0)=\varphi
$$

and $f_{j}$ is defined by

$$
f_{j}(u)=\pi\left(V(t) u_{0, j}, u\right)-\pi(u, u)+g(u) .
$$

We intend to show that there exists a nondecreasing polynomial function $P \geq 1$ such that

$$
\begin{aligned}
\left\|u_{1}-u_{2}\right\|_{\dot{Z}_{T}} \lesssim P\left(\left\|u_{1}\right\|_{\dot{Z}_{T}}+\left\|u_{2}\right\|_{\dot{Z}_{T}}\right)\left[\left\|u_{0,1}-u_{0,2}\right\|_{\dot{H}^{s} k}\right. \\
\left.+\left(\left\|u_{1}\right\|_{\dot{X}^{s_{k}}}+\left\|u_{2}\right\|_{\dot{X}^{s_{k}}}\right)\left\|u_{1}-u_{2}\right\|_{\dot{Z}_{T}}\right]
\end{aligned}
$$

where the implicit constant in the inequality may depends on $u_{0,1}, u_{0,2}$. Obviously, the uniqueness of the solution to (gBO and the fact that the flow map is locally Lipschitz from $\dot{H}^{s_{k}}(\mathbb{R})$ to $Z_{T}$ follow directly from (4.2).

One has

$$
\left\|U_{1}(t) u_{0,1}-U_{2}(t) u_{0,2}\right\|_{\dot{Z}_{T}} \lesssim\left\|U_{1}(t)\left(u_{0,1}-u_{0,2}\right)\right\|_{\dot{Z}_{T}}+\left\|\left(U_{1}(t)-U_{2}(t)\right) u_{0,2}\right\|_{\dot{Z}_{T}} .
$$

The first term in the right-hand side is bounded by $\left\|u_{0,1}-u_{0,2}\right\|_{\dot{H}^{s_{k}}}$. To treat the second one, we note that $\left(U_{1}(t)-U_{2}(t)\right) u_{0,2}$ is solution to

$$
\partial_{t} u+\mathcal{H} \partial_{x}^{2} u+\pi\left(V(t) u_{0,1}, u\right)=\pi\left(V(t) u_{0,1}, U_{2}(t) u_{0,2}\right)-\pi\left(V(t) u_{0,2}, U_{2}(t) u_{0,2}\right)
$$


with zero initial data. Hence by Propositions 3.2 and 3.3 ,

$$
\begin{aligned}
\left\|\left(U_{1}(t)-U_{2}(t)\right) u_{0,2}\right\|_{\dot{Z}_{T}} & \lesssim\left\|\pi\left(V(t) u_{0,1}, U_{2}(t) u_{0,2}\right)-\pi\left(V(t) u_{0,2}, U_{2}(t) u_{0,2}\right)\right\|_{\dot{\mathcal{N}}^{s_{k}}, 1} \\
& \lesssim\left\|u_{0,1}-u_{0,2}\right\|_{\dot{H}^{s_{k}}} .
\end{aligned}
$$

We also need to bound

$$
\begin{aligned}
& \left\|\int_{0}^{t}\left(U_{1}\left(t-t^{\prime}\right) f_{1}\left(u_{1}\right)-U_{2}\left(t-t^{\prime}\right) f_{2}\left(u_{2}\right)\right) d t^{\prime}\right\|_{\dot{Z}_{T}} \\
& \lesssim\left\|\int_{0}^{t} U_{1}\left(t-t^{\prime}\right)\left(f_{1}\left(u_{1}\right)-f_{1}\left(u_{2}\right)\right) d t^{\prime}\right\|_{\dot{Z}_{T}} \\
& \quad+\left\|\int_{0}^{t} U_{1}\left(t-t^{\prime}\right)\left(f_{1}\left(u_{2}\right)-f_{2}\left(u_{2}\right)\right) d t^{\prime}\right\|_{\dot{Z}_{T}} \\
& \quad+\left\|\int_{0}^{t}\left(U_{1}\left(t-t^{\prime}\right)-U_{2}\left(t-t^{\prime}\right)\right) f_{2}\left(u_{2}\right) d t^{\prime}\right\|_{\dot{Z}_{T}} .
\end{aligned}
$$

(4.3) is bounded by

$$
(4.3) \lesssim\left\|f_{1}\left(u_{1}\right)-f_{1}\left(u_{2}\right)\right\|_{\dot{\mathcal{N}}^{s}, 1}
$$

and we can use (4.1) to get the desired estimate. Term (4.4) is bounded by

$$
\begin{aligned}
\text { (4.4) } & \lesssim\left\|\pi\left(V(t) u_{0,1}, u_{2}\right)-\pi\left(V(t) u_{0,2}, u_{2}\right)\right\|_{\dot{\mathcal{N}}^{s_{k}}, 1} \\
& \lesssim\left\|u_{2}\right\|_{\dot{X}^{s_{k}}}\left\|u_{0,1}-u_{0,2}\right\|_{\dot{H}^{s_{k}}} .
\end{aligned}
$$

Finally, note that $\int_{0}^{t}\left(U_{1}\left(t-t^{\prime}\right)-U_{2}\left(t-t^{\prime}\right)\right) f_{2}\left(u_{2}\right) d t^{\prime}$ is solution to

$$
\partial_{t} u+\mathcal{H} \partial_{x}^{2} u+\pi\left(V(t) u_{0,1}, u\right)=\pi\left(V(t) u_{0,2}, \psi\right)-\pi\left(V(t) u_{0,1}, \psi\right),
$$

with zero initial data, and where $\psi=\int_{0}^{t} U_{2}\left(t-t^{\prime}\right) f_{2}\left(u_{2}\right) d t^{\prime}$. It follows that

$$
\begin{aligned}
\text { (4.5) } & \lesssim\left\|\pi\left(V(t) u_{0,2}, \psi\right)-\pi\left(V(t) u_{0,1}, \psi\right)\right\|_{\dot{\mathcal{N}}^{s_{k}}, 1} \\
& \lesssim\|\psi\|_{\dot{X}^{s_{k}}}\left\|u_{0,1}-u_{0,2}\right\|_{\dot{H}^{s_{k}}} \\
& \lesssim\left(\left\|u_{2}\right\|_{\dot{X}^{s_{k}}}+\left\|u_{2}\right\|_{\dot{X}^{s_{k}}}^{k+1}\right)\left\|u_{0,1}-u_{0,2}\right\|_{\dot{H}^{s_{k}}} .
\end{aligned}
$$

Gathering all these estimates we obtain (4.2).

\subsection{Existence in $H^{s}(\mathbb{R}), s \geq s_{k}$}

Define the spaces $X^{s}=\dot{X}^{0} \cap \dot{X}^{s}$ and $\mathcal{N}^{s, \theta}=\dot{\mathcal{N}}^{0, \theta} \cap \dot{\mathcal{N}}^{s, \theta}$. 
We closely follow the proof of Theorem 2.1. We show that $F$ is a contraction in the intersection of

$$
B_{M}\left(u_{0}, T\right)=\left\{u \in X^{s} \cap L_{x}^{k} L_{T}^{\infty}:\left\|u-u_{0}\right\|_{L_{x}^{k} L_{T}^{\infty}} \leq \delta\right\}
$$

and

$$
B_{S}\left(u_{0}, T\right)=\left\{u \in X^{s} \cap L_{x}^{k} L_{T}^{\infty}:\|u\|_{X^{s}} \leq \delta\right\}
$$

endowed with the norm

$$
\|u\|_{Y_{T}}=\|u\|_{X^{s}}+\|u\|_{L_{x}^{k} L_{T}^{\infty}} .
$$

Using Propositions 3.2, 3.3 and 4.1 (applied with $s \geq s_{k}$ and $s=0$ ) and the embedding $\mathcal{N}^{s, 1} \hookrightarrow \dot{\mathcal{N}}^{s_{k}, 1}$ for $s \geq s_{k}$ we find

$$
\begin{aligned}
\|F(u)\|_{X^{s}} & \leq C\left\|U(t) u_{0}\right\|_{X^{s}}+C\left(1+\left\|u-u_{0}\right\|_{L_{x}^{k} L_{T}^{\infty}}^{k-1}\right)\|u\|_{X^{s}}^{2} \\
& +C\left(\left\|u_{L}-u_{0}\right\|_{L_{x}^{k} L_{T}^{\infty}}+\left\|u-u_{0}\right\|_{L_{x}^{k} L_{T}^{\infty}}\right)\left(1+\left\|u-u_{0}\right\|_{L_{x}^{k} L_{T}^{\infty}}^{k-1}\right)\|u\|_{X^{s}}
\end{aligned}
$$

and

$$
\begin{aligned}
\| F(u) & -u_{0}\left\|_{L_{x}^{k} L_{T}^{\infty}} \leq\right\| U(t) u_{0}-u_{0}\left\|_{L_{x}^{k} L_{T}^{\infty}}+C\left(1+\left\|u-u_{0}\right\|_{L_{x}^{k} L_{T}^{\infty}}^{k-1}\right)\right\| u \|_{X^{s}}^{2} \\
& +C\left(\left\|u_{L}-u_{0}\right\|_{L_{x}^{k} L_{T}^{\infty}}+\left\|u-u_{0}\right\|_{L_{x}^{k} L_{T}^{\infty}}\right)\left(1+\left\|u-u_{0}\right\|_{L_{x}^{k} L_{T}^{\infty}}^{k-1}\right)\|u\|_{X^{s}}
\end{aligned}
$$

In the same way, one may show that

$$
\begin{aligned}
\left\|F\left(u_{1}\right)-F\left(u_{2}\right)\right\|_{Y_{T}} \lesssim & \left\|f\left(u_{1}\right)-f\left(u_{2}\right)\right\|_{\mathcal{N}^{s, 1}} \\
\lesssim & \left\|u_{L}-u_{1}\right\|_{L_{x}^{k} L_{T}^{\infty}}\left(1+\left\|u_{1}\right\|_{L_{x}^{k} L_{T}^{\infty}}^{k-1}\right)\left\|u_{1}-u_{2}\right\|_{X^{s}} \\
& +\left\|u_{2}\right\|_{X^{s}}\left(\left\|u_{1}\right\|_{L_{x}^{k} L_{T}^{\infty}}^{k-1}+\left\|u_{2}\right\|_{L_{x}^{k} L_{T}^{\infty}}^{k-1}\right)\left\|u_{1}-u_{2}\right\|_{L_{x}^{k} L_{T}^{\infty}} \\
& +\left\|u_{1}\right\|_{X^{s}}^{2}\left(\left\|u_{1}\right\|_{L_{x}^{k} L_{T}^{\infty}}^{k-2}+\left\|u_{2}\right\|_{L_{x}^{k} L_{T}^{\infty}}^{k-2}\right)\left\|u_{1}-u_{2}\right\|_{L_{x}^{k} L_{T}^{\infty}} \\
& +\left\|u_{2}\right\|_{L_{x}^{k} L_{T}^{\infty}}^{k-1}\left(\left\|u_{1}\right\|_{X^{s}}+\left\|u_{2}\right\|_{X^{s}}\right)\left\|u_{1}-u_{2}\right\|_{X^{s}} .
\end{aligned}
$$

This proves the existence in $H^{s}(\mathbb{R})$. The end of the proof is identical to that of Theorem 2.1

\section{Well-posedness for $k=3$}

Let $k=3$ and $s>1 / 3$ be fixed.

The scheme of the proof is the same as for the case $k \geq 4$ with minor modifications. First, in view of Lemma 3.2, it is clear that Lemma 3.5 holds 
for $k=3$ with $u_{0} \in \dot{H}^{s_{k}}$ replaced by $u_{0} \in H^{s}$. Next we see that the $\dot{\mathcal{B}}_{\left(\frac{1}{k}-\frac{\varepsilon}{4}\right)^{-1}}^{\frac{3 \varepsilon}{4}, 2}\left(L_{T}^{\frac{2}{\varepsilon}}\right)$-norm which appears in Proposition 4.1 when estimating the nonlinear term $g$ is not bounded by the $\dot{S}^{\varepsilon, 1}$-norm for $k=3$. So we modify slightly the space $X^{s}$ by setting

$$
X^{s}=\dot{X}^{0} \cap \dot{X}^{s} \cap \dot{\mathcal{B}}_{3}^{\varepsilon, 2}\left(L_{T}^{\frac{2}{\varepsilon}}\right) .
$$

On one hand, it is clear from Sobolev inequalities that

$$
\|u\|_{\dot{\mathcal{B}}_{\left(\frac{1}{3}-\frac{3 \varepsilon}{4}\right)^{-1}}\left(L_{T}^{\left.\frac{2}{\varepsilon}\right)}\right.} \lesssim\|u\|_{\dot{\mathcal{B}}_{3}^{\varepsilon, 2}\left(L_{T}^{\frac{2}{\varepsilon}}\right)} \lesssim\|u\|_{X^{s}}
$$

On the other hand, the $\dot{\mathcal{B}}_{3}^{\varepsilon, 2}\left(L_{T}^{\frac{2}{\varepsilon}}\right)$-norm is acceptable since by (3.8),

$$
\begin{aligned}
\|V(t) \varphi\|_{\dot{\mathcal{B}}_{3}^{\varepsilon, 2}\left(L_{T}^{\frac{2}{\varepsilon}}\right)} \lesssim\left(\sum_{j} 4^{j \varepsilon}\left\|Q_{j} V(t) \varphi\right\|_{L_{x}^{3} L_{T}^{\infty}}^{2}\right)^{1 / 2} & \\
& \lesssim\left(\sum_{j}\left\|Q_{j} \varphi\right\|_{H^{1 / 3+2 \varepsilon}}^{2}\right)^{1 / 2} \lesssim\|\varphi\|_{H^{s}}
\end{aligned}
$$

for $\varepsilon \ll 1$. From this, it is straightforward to check that the subcritical nonhomogeneous versions of Propositions 3.2, 3.3 and 4.1 are valid whenever $k=3$. This essentially proves Theorem 2.3

\section{Acknowledgments}

The author wants to thank Fabrice Planchon for his enthusiastic help and his availability.

\section{References}

[1] T. B. Benjamin. Internal waves of permanent form in fluids of great depth. Journal of Fluid Mechanics, 29:559-592, 1967.

[2] H. A. Biagioni and F. Linares. Ill-posedness for the derivative Schrödinger and generalized Benjamin-Ono equations. Trans. Amer. Math. Soc., 353(9):3649-3659 (electronic), 2001.

[3] N. Burq and F. Planchon. Smoothing and dispersive estimates for 1D Schrödinger equations with BV coefficients and applications. J. Funct. Anal., 236(1):265-298, 2006. 
[4] N. Burq and F. Planchon. On well-posedness for the Benjamin-Ono equation. Math. Ann., 340(3):497-542, 2008.

[5] A. D. Ionescu and C. E. Kenig. Global well-posedness of the BenjaminOno equation in low-regularity spaces. J. Amer. Math. Soc., 20(3):753798 (electronic), 2007.

[6] R. J. Iório, Jr. On the Cauchy problem for the Benjamin-Ono equation. Comm. Partial Differential Equations, 11(10):1031-1081, 1986.

[7] C. E. Kenig, G. Ponce, and L. Vega. Oscillatory integrals and regularity of dispersive equations. Indiana Univ. Math. J., 40(1):33-69, 1991.

[8] C. E. Kenig, G. Ponce, and L. Vega. Well-posedness of the initial value problem for the Korteweg-de Vries equation. J. Amer. Math. Soc., 4(2):323-347, 1991.

[9] C. E. Kenig, G. Ponce, and L. Vega. Well-posedness and scattering results for the generalized Korteweg-de Vries equation via the contraction principle. Comm. Pure Appl. Math., 46(4):527-620, 1993.

[10] C. E. Kenig and H. Takaoka. Global wellposedness of the modified Benjamin-Ono equation with initial data in $H^{1 / 2}$. Int. Math. Res. Not., pages Art. ID 95702, 44, 2006.

[11] H. Koch and N. Tzvetkov. Nonlinear wave interactions for the Benjamin-Ono equation. Int. Math. Res. Not., (30):1833-1847, 2005.

[12] L. Molinet and F. Ribaud. Well-posedness results for the generalized Benjamin-Ono equation with arbitrary large initial data. Int. Math. Res. Not., (70):3757-3795, 2004.

[13] L. Molinet and F. Ribaud. Well-posedness results for the generalized Benjamin-Ono equation with small initial data. J. Math. Pures Appl. (9), 83(2):277-311, 2004.

[14] L. Molinet, J.-C. Saut, and N. Tzvetkov. Ill-posedness issues for the Benjamin-Ono and related equations. SIAM J. Math. Anal., 33(4):982988 (electronic), 2001.

[15] H. Ono. Algebraic solitary waves in stratified fluids. J. Phys. Soc. Japan, 39(4):1082-1091, 1975.

[16] G. Ponce. On the global well-posedness of the Benjamin-Ono equation. Differential Integral Equations, 4(3):527-542, 1991. 
[17] J.-C. Saut. Sur quelques généralisations de l'équation de Korteweg-de Vries. J. Math. Pures Appl. (9), 58(1):21-61, 1979.

[18] T. Tao. Global well-posedness of the Benjamin-Ono equation in $H^{1}(\mathbf{R})$. J. Hyperbolic Differ. Equ., 1(1):27-49, 2004.

[19] S. Vento. Sharp well-posedness results for the generalized BenjaminOno equation with high nonlinearity, 2007. 